\title{
Expert opinion on best practice guidelines and competency framework for visual screening in children
}

September 2013, Vol. 21, No. 3 , Pages 169-174 (doi:10.3109/09273972.2013.811603)

Carla Lança, MSc

Escola Superior de Tecnologia da Saúde de Lisboa, Centro de Investigação e Estudos em Saúde Pública (CIESP), Escola Nacional de Saúde Pública.

Correspondence:

Carla Lança, E-mail: carla.costa@estesl.ipl.pt

\section{Abstract}

Purpose: Screening programs to detect visual abnormalities in children vary among countries. The aim of this study is to describe experts' perception of best practice guidelines and competency framework for visual screening in children.

Methods: A qualitative focus group technique was applied during the Portuguese national orthoptic congress to obtain the perception of an expert panel of 5 orthoptists and 2 ophthalmologists with experience in visual screening for children (mean age 53.43 years, $S D \pm 9.40$ ). The panel received in advance a script with the description of three tuning competencies dimensions (instrumental, systemic, and interpersonal) for visual screening. The session was recorded in video and audio. Qualitative data were analyzed using a categorical technique.

Results: According to experts' views, six tests (35.29\%) have to be included in a visual screening: distance visual acuity test, cover test, bi-prism or $4 / 6^{\Delta}$ prism, fusion, ocular movements, and refraction. Screening should be performed according to the child age before and after 3 years of age (17.65\%). The expert panel highlighted the influence of the professional experience in the application of a screening protocol (23.53\%). They also showed concern about the false negatives control (23.53\%). Instrumental competencies were the most cited $(54.09 \%)$, followed by interpersonal $(29.51 \%)$ and systemic (16.4\%). 
Conclusions: Orthoptists should have professional experience before starting to apply a screening protocol. False negative results are a concern that has to be more thoroughly investigated. The proposed framework focuses on core competencies highlighted by the expert panel. Competencies programs could be important do develop better screening programs.

\section{Keywords}

Allied health, education (medical), orthoptists, public health/policy/standards, screening and infant vision development, strabismus

Read More: http://informahealthcare.com/doi/abs/10.3109/09273972.2013.811603 